\title{
Some conjectures for cogwheel phase cycling
}

\author{
Colan E. Hughes, ${ }^{\mathrm{a}}$ Marina Carravetta, ${ }^{\mathrm{b}}$ and Malcolm H. Levitt ${ }^{\mathrm{b}, *}$ \\ a Department of NMR Based Structural Biology, Max-Planck-Institute for Biophysical Chemistry, Am Faßberg 11, 37077 Göttingen, Germany \\ ${ }^{\mathrm{b}}$ Department of Chemistry, University of Southampton, Southampton SO17 1BJ, UK
}

Received 30 October 2003; revised 7 January 2004

\begin{abstract}
Cogwheel phase cycles are often significantly shorter than traditional nested phase cycles. However, optimal solutions for cogwheel cycles are often difficult to find. This paper presents techniques and conjectures which the authors have found useful for generating cogwheel phase cycles either without the need for computer searches or with significantly smaller searches than would otherwise be necessary. The conjectures presently lack proofs but have been tested successfully for a large number of cases.

(c) 2004 Elsevier Inc. All rights reserved.
\end{abstract}

\section{Introduction}

Cogwheel phase cycles [1] are frequently much shorter than traditional "nested" phase cycles $[2,3]$ but are often more difficult to generate. Some optimum cogwheel phase cycles can be predicted using cogwheel selection diagrams but, in general, computer searches must be used to find the shortest cogwheel phase cycle which achieves a particular coherence pathway selectivity. With savings in phase-cycle length of $34 \%$ achieved for 3QMAS [1] and 85\% achieved for the TOSS experiment with $5 \pi$ pulses [4], the benefits are clear, but without relatively straightforward determination of optimal cycles, cogwheel phase cycling will always be difficult to implement. Computer programs have been written for simulating the selectivity of phase cycles [5] and for searching for suitable cogwheel phase cycles [6]. However, without a good estimate of the shortest possible length of the phase cycle, long search times may be necessary.

To address this problem, we have looked for patterns in the shortest lengths of cogwheel phase cycles for different coherence pathway selection tasks. From these patterns, we have developed predictive equations which, in certain cases, allow the optimal cogwheel cycle to be written down immediately. We are also able to identify

\footnotetext{
${ }^{*}$ Corresponding author. Fax: +44-23-8059-3781.

E-mail address: mhl@soton.ac.uk (M.H. Levitt).
}

the conditions under which this prediction fails. Even in these cases, it is possible to place a reliable lower bound on the length of the optimal cogwheel phase cycle, which greatly improves the speed of numerical searches.

\section{Coherence selection}

An NMR experiment typically involves a sequence of RF irradiation blocks, each of which induces coherence transfers. The NMR signal is a superposition of components, each of which has a different history of coherence orders. This history of orders is called the coherence transfer pathway [2]. The relative phases of $\mathrm{RF}$ irradiation elements are fixed within each RF block, but the overall phases of the different RF blocks may be varied with respect to each other. In phase cycling, the overall phases are varied in a cyclic strategy so as to select out signal components deriving from one or more coherence transfer pathways, with other pathways suppressed exactly.

Suppose that there are $n$ RF blocks in the sequence, numbered 1 to $n$. The interval before block $l$ is denoted $\left\{l^{-}\right\}$, while the interval after block $l$ is denoted $\left\{l^{+}\right\}$. By definition

$\left\{l^{-}\right\}=\left\{(l-1)^{+}\right\}$.

An individual coherence pathway is denoted

$\boldsymbol{p}=\left\{p_{0^{+}}, p_{1^{+}}, p_{2^{+}}, \ldots, p_{n^{+}}\right\}$. 
The change in coherence order occurring at block $l$ may be defined as

$\Delta p_{l}=p_{(l-1)^{+}}-p_{l^{+}}$.

The desired coherence pathways are denoted by the superscripts $0,0^{\prime}, 0^{\prime \prime}$, etc. This gives $\boldsymbol{p}^{0}=\left\{p_{0^{+}}^{0}, p_{1^{+}}^{0}, p_{2^{+}}^{0}, \ldots\right.$, $\left.p_{n^{+}}^{0}\right\}, \boldsymbol{p}^{0^{\prime}}=\left\{p_{0^{+}}^{0^{\prime}}, p_{1^{+}}^{0^{\prime}}, p_{2^{+}}^{0^{\prime}}, \ldots, p_{n^{+}}^{0^{\prime}}\right\}$, etc. The maximum coherence order possible in the interval between two RF blocks is denoted $p_{l^{+}}^{\max }$ and the minimum is denoted $p_{l^{+}}^{\min }$. It is assumed that all coherence orders between $p_{l^{+}}^{\min }$ and $p_{l^{+}}^{\max }$ need to be taken into account. In what follows, it is the case that $p_{l^{+}}^{\min }=-p_{l^{+}}^{\max }$. Examples can be envisaged in which both of these conditions are relaxed.

A phase cycle will produce a number of transients which are added to generate the final signal. For the transient $m$, the phase of the RF block $l$ is denoted $\phi_{l}^{(m)}$. The accumulated phase for a particular coherence pathway, $\boldsymbol{p}$, and transient, $m$, is $\Phi^{m}(\boldsymbol{p})$, given by

$\Phi^{(m)}(\boldsymbol{p})=\sum_{l=1}^{n} \phi_{l}^{(m)} \Delta p_{l}+\phi_{\mathrm{sig}}^{(m)}$,

where $\phi_{\text {sig }}^{(m)}$ is the signal phase shift, implemented either by a RF phase shift of the receiver reference wave or by post-digitization data processing (receiver imperfections are ignored [4]). The selectivity of a phase cycle may be summarized as

$$
\begin{aligned}
N^{-1} \sum_{m=0}^{N-1} \exp \left\{-i \Phi^{(m)}(\boldsymbol{p})\right\} & =1 \quad \text { if } \boldsymbol{p} \in\left\{\boldsymbol{p}^{0}, \boldsymbol{p}^{0^{\prime}}, \ldots\right\}, \\
& =0 \quad \text { otherwise, }
\end{aligned}
$$

where $\left\{\boldsymbol{p}^{0}, \boldsymbol{p}^{0^{\prime}}, \ldots\right\}$ is the set of desired coherence pathways and the sum is over all the transients in the phase cycle. The necessary constructive and destructive interference for Eq. (5) to hold will be achieved if $\Phi^{(m)}(\boldsymbol{p})$ is zero or an integer multiple of $2 \pi$ for all transients only when $\boldsymbol{p}$ is one of the desired coherence pathways, i.e.,

$$
\begin{aligned}
& \Phi^{(m)}(\boldsymbol{p})=2 \pi Z \quad \text { if } \boldsymbol{p} \in\left\{\boldsymbol{p}^{0}, \boldsymbol{p}^{0^{\prime}}, \ldots\right\}, \\
& \neq 2 \pi Z \text { otherwise, }
\end{aligned}
$$

where $Z$ is any integer.

In what follows, it is convenient to define everything with respect to the intervals between the RF blocks, rather than to the blocks themselves. The difference between the phases of adjacent RF blocks is notated

$\Delta \phi_{l^{+}}^{(m)}=\phi_{l+1}^{(m)}-\phi_{l}^{(m)}$.

The first coherence order, $p_{0^{+}}$, can only be zero, whilst, assuming a perfect receiver, the last, $p_{n^{+}}$, can only be -1 . Therefore, Eqs. (3), (4), and (7) can be combined to give

$\Phi^{(m)}(\boldsymbol{p})=-\sum_{l=1}^{n} \Delta \phi_{l^{+}}^{(m)} p_{l^{+}}$,

with the definition $\Delta \phi_{n^{+}}^{(m)}=\phi_{\mathrm{sig}}^{(m)}-\phi_{n}^{(m)}$.

\section{Nested phase cycling}

In nested phase cycling, the phase of a particular RF block is cycled whilst keeping the phase all other RF blocks constant. The phase of a second block is then incremented by one step in its cycle and the first cycle is repeated. When the second cycle is completed, a third cycle is begun, with the second and first cycles fully repeated. In this way, the selection of desired coherence pathways is ensured for each part of the pulse sequence.

A sequence of two RF blocks has one coherence order, $p_{1^{+}}$, which may vary, since $p_{0^{+}}=0$ and $p_{2^{+}}=-1$. Selection of a particular value, $p_{1^{+}}^{0}$, requires a phase cycle, on either block, of length $p_{1^{+}}^{\max }+1+\left|p_{1^{+}}^{0}\right|$, following the procedure of Bodenhausen et al. [2]. A sequence of three RF blocks has two variable coherence orders and selection of a single pathway $\left\{0, p_{1^{+}}^{0}, p_{2^{+}}^{0},-1\right\}$ requires a cycle of $p_{1^{+}}^{\max }+1+\left|p_{1^{+}}^{0}\right|$ steps on block 1 or 2 and a second cycle of $p_{2^{+}}^{\max }+1+\left|p_{2^{+}}^{0}\right|$ on block 2 or 3 , noting that these cycles cannot both be on block 2 . This method may be continued for sequences with any number of RF blocks. Selection of $p_{l^{+}}^{0}$ requires a cycle of $p_{l^{+}}^{\max }+1+\left|p_{l^{+}}^{0}\right|$ steps on either block $l$ or block $l+1$. Thus, the total cycle length for a single coherence order pathway will be the product of the cycles corresponding to each selected coherence order, given by

$N_{\text {nest }}=\prod_{l=1}^{n-1}\left(p_{l^{+}}^{\max }+1+\left|p_{l^{+}}^{0}\right|\right)$,

where the product is over the $n-1$ intervals between the $\mathrm{RF}$ blocks in the experiment. If the cycle with length $p_{l^{+}}^{\max }+1+\left|p_{l^{+}}^{0}\right|$ is applied to the block $l$ (i.e., the RF block preceding the interval during which $p_{l^{+}}^{0}$ is selected), then the phase increment for block $l, \phi_{l}^{\text {inc }}$, is given by

$\phi_{l}^{\mathrm{inc}}=\frac{2 \pi}{p_{l^{+}}^{\max }+1+\left|p_{l^{+}}^{0}\right|}$.

The signal phase shift is given by

$\phi_{\text {sig }}^{(m)}=-\sum_{l=1}^{n} \phi_{l}^{(m)} \Delta p_{l}^{0}$,

where $\Delta p_{l}^{0}=p_{l^{+}}^{0}-p_{l-1^{+}}^{0}$. Inserting Eq. (11) into Eq. (4) gives zero, ensuring that the pathway is selected, whilst the choices of $\phi_{l}^{\text {inc }}$ ensure that no other coherence pathways fulfil the selection in Eq. (5). As noted elsewhere [7], this simple method can lead to phase cycles with many redundant steps.

\section{Cogwheel phase cycling}

For cogwheel phase cycles, the phases of all the RF blocks are cycled at the same time. The cogwheel phase cycle is given by several parameters. There is the length of the cycle, $N$, and there is the set of winding numbers 
for each RF block, denoted $v_{l}$. These give the increment in the phase of each RF block for successive transients

$\phi_{l}^{\text {inc }}=\frac{2 \pi v_{l}}{N}$.

Because all phases are incremented together, the phase of each RF block at each successive acquisition is given by

$\phi_{l}^{(m)}=\frac{2 \pi v_{l}}{N} m$,

where $m$ is the phase-cycle counter, taking values from 0 to $N-1$. There is also a winding number for the signal phase shift. This is referred to as the signal winding number, $v_{\text {sig }}$, and is defined by

$\phi_{\text {sig }}^{(m)}=\frac{2 \pi v_{\text {sig }}}{N} m$.

It is also convenient to define nomenclature for the difference between winding numbers for successive RF blocks. These are denoted $\Delta v_{l^{+}}$and correspond to the interval following block $l$. They are related to the winding numbers by

$\Delta v_{l^{+}}=v_{l+1}-v_{l}$.

As with $\Delta \phi_{n^{+}}^{(m)}$, the value of $\Delta v_{n^{+}}$is defined as

$\Delta v_{n^{+}}=v_{\text {sig }}-v_{n}$.

The nomenclature is summarized in Fig. 1. From Eq (8), the accumulated phase is therefore given by

$\Phi^{(m)}(\boldsymbol{p})=-\frac{2 \pi m}{N} \sum_{l=1}^{n} \Delta v_{l^{+}} p_{l^{+}}$,

with respect to the winding numbers.

Cogwheel phase cycles can be represented as $\operatorname{COG} N\left(v_{1}, v_{2}, \ldots, v_{n} ; v_{\text {sig }}\right)$. In what follows, predictions will be presented for the optimal values of $N$ and $\Delta v_{l^{+}}$ which correspond to the shortest possible cogwheel phase cycles. To convert from such a set of values to a representation of the form $\operatorname{COGN}\left(v_{1}, v_{2}, \ldots, v_{n} ; v_{\text {sig }}\right)$, the following procedure can be used. First, decide upon a value for $v_{1}$, which can be anything (including zero). From this starting point, the equation
$v_{l+1}=v_{l}+\Delta v_{l^{+}}$,

can be used to generate the values of $v_{2}, v_{3}, \ldots, v_{n}$. For constructive interference of the signals, the value of $\Phi^{(m)}(\boldsymbol{p})$ must satisfy Eq. (6). Setting all $\Phi^{(m)}\left(\boldsymbol{p}^{0}\right)$ to zero allows Eq. (17) to be rearranged (noting that $p_{n^{+}}=-1$ ) to give

$\Delta v_{n^{+}}=\sum_{l=1}^{n-1} \Delta v_{l^{+}} p_{l^{+}}$

This expression can be combined with Eq. (16) to give

$v_{\text {sig }}=v_{n}+\sum_{l=1}^{n-1} \Delta v_{l^{+}} p_{l^{+}}$

which can be used to calculate $v_{\text {sig. }}$.

\section{Numerical searches}

Unlike nested phase cycles, there do not exist simple formulae, such as Eqs. (9) and (10), for the length and phase increments of cogwheel phase cycles, hence the reliance up to now on numerical searches. For a cycle of length $N$ there will be $N-1$ possible values for each winding number, running from 1 to $N-1$. Because the winding numbers relate to phases (Eq. (13)), this range is equivalent to $-N / 2$ to $N / 2-1$ for even $N$ and $-(N-1) / 2$ to $(N-1) / 2$ for odd $N$, with all zeroes excluded. If there are $n$ RF blocks, $n-1$ blocks will need to be phase cycled and the number of possible combinations of winding numbers will therefore be $(N-1)^{n-1}$. As a first step, we address the problem of very large searches by looking for rules which tell us which of the possible combinations of winding numbers will be equivalent, in order to reduce the search area.

The accumulated phase for a particular coherence pathway, $\boldsymbol{p}$, and transient, $m$, in an experiment using a cogwheel phase cycle is given by Eq. (17). If the pathway is to be selected by the phase cycle, $\Phi^{(m)}(\boldsymbol{p})$ must fulfil Eq. (6) for all values of $m$. Hence

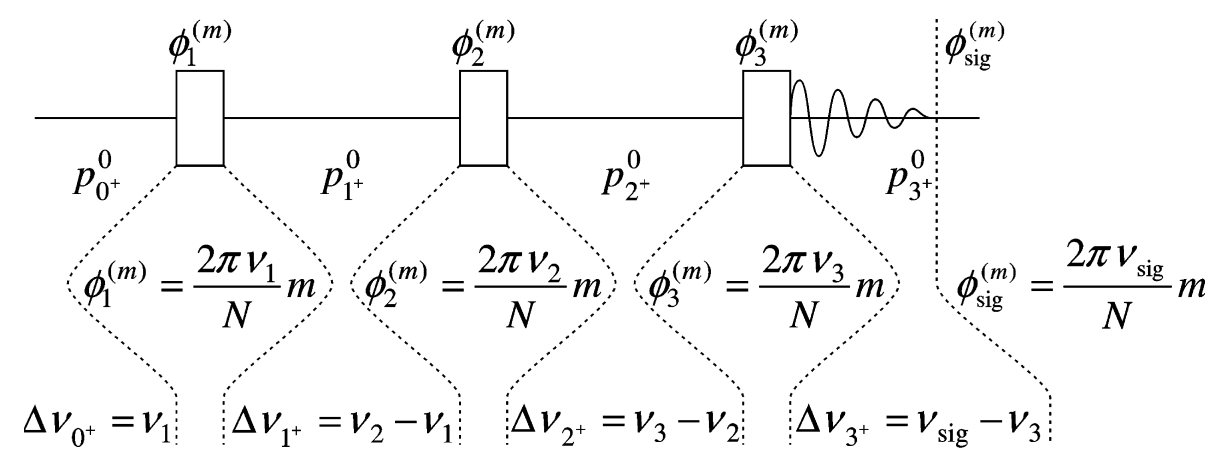

Fig. 1. Nomenclature for coherence orders, phases, and winding numbers. A three-block pulse sequence is illustrated, i.e., $n=3$. The parameters $\phi_{l}^{(m)}$ and $v_{l}$ pertain to the RF block $l$, whilst $p_{l^{+}}^{0}$ and $\Delta v_{l^{+}}$pertain to the interval following the RF block $l$. 


$$
\begin{aligned}
\sum_{l=1}^{n} \Delta v_{l^{+}} p_{l^{+}} & =Z N \quad \text { if } \boldsymbol{p} \in\left\{\boldsymbol{p}^{0}, \boldsymbol{p}^{0^{\prime}}, \ldots\right\} \\
& \neq Z N \quad \text { otherwise }
\end{aligned}
$$

where $Z$ is any integer. This can be rewritten as

$$
\begin{aligned}
\left(\sum_{l=1}^{n} \Delta v_{l^{+}} p_{l^{+}}\right) \bmod N & =0 \quad \text { if } \boldsymbol{p} \in\left\{\boldsymbol{p}^{0}, \boldsymbol{p}^{0^{\prime}}, \ldots\right\}, \\
& \neq 0 \quad \text { otherwise. }
\end{aligned}
$$

This form of the selection rule has been found to be the most amenable in computer searches for optimal cogwheel phase cycles. From this selection rule, the following points can be noted which limit the search area and indicate coherence pathways which have related selectivities by cogwheel phase cycles.

1. The sign of all winding numbers may be reversed without changing the selectivity of the phase cycle. Thus, the value of one $\Delta v_{l^{+}}$can be limited to the range 1 to $+N / 2$.

2. A cogwheel phase cycle based upon values $\left\{N, c \Delta v_{1^{+}}\right.$, $\left.c \Delta v_{2^{+}}, c \Delta v_{3^{+}}, \ldots\right\}$ will have the same selectivity as one based upon the values $\left\{N / d, \Delta v_{1^{+}}, \Delta v_{2^{+}}, \Delta v_{3^{+}}, \ldots\right\}$, where $d$ is the greatest common denominator of $N$ and $c$. Thus, any phase cycle in which the values of $\Delta v_{l^{+}}$all share a common integer factor may be dropped from the search, as they correspond to other phase cycles with smaller winding numbers and, in when $d \neq 1$, fewer transients.

3. The optimal cogwheel phase-cycle length for selecting a single pathway is independent of the signs of the selected coherence orders. A cycle which selects a given single coherence pathway can be made selective for another pathway which differs only in sign by changing the signs of the winding numbers $\Delta v_{l^{+}}$corresponding to those coherence orders which have changed sign.

Point 1 stems from the fact that if $x \bmod N=0$, $-x \bmod N=0$ as well and therefore, from Eq. (22), the overall sign of the accumulated phase (and hence of the winding numbers) is irrelevant. Point 2 stems from the nature of the $\bmod$ function. If $c x \bmod N=0$, then if $d$ is the greatest common denominator of $c$ and $N, x \bmod N / d$ $=0$, whilst if $c y \bmod N \neq 0$, then $y \bmod N / d \neq 0$ as well. Point 3 comes from the fact that by changing the sign of both $\Delta v_{l^{+}}$and $p_{l^{+}}$, nothing in the selection rule equation changes. This last point removes the need for separate calculation of phase cycles for different coherence order pathways related by sign changes.

Taking these points into account still leaves a great many possible phase cycles which must be tested for any given cycle length, $N$. The first point reduces this number to $\frac{1}{2}\left((N-1)^{n-1}\right)$. The second point also reduces this number significantly but, as the reduction depends upon the factorization of the winding numbers (which is related to the distribution of prime numbers), this cannot easily be calculated, although the reduction declines as the number of RF blocks increases. Searching for optimal cogwheel phase cycles can be very lengthy, even with the reductions in search area mentioned above. With this in mind, the following conjectures predicting the cogwheel parameters are presented. They include a starting value of $N$ for numerical searches.

\section{Predictive formulae}

\subsection{Selection of a single coherence pathway}

We have examined the optimal $N$ values for a large number of cogwheel solutions, discovered by extensive numerical searches. Starting with the case of $n=3$ (selecting two coherence orders), then $n=4$ (selecting three coherence orders), we have identified patterns which either allow the optimal cogwheel cycle to be predicted immediately or allow useful constraints to be placed on numerical searches for the optimal cogwheel parameters. From these patterns, we have constructed general patterns for any value of $n$. Consider the following quantities:

$N_{\text {pred }}=Q+\sum_{l=1}^{n-1}\left(\frac{2 Q\left|p_{l^{+}}^{0}\right|}{q_{l^{+}}}\right)$,

$\xi_{l^{+}}=\frac{\operatorname{sgn}\left(p_{l^{+}}^{0}\right) Q}{q_{l^{+}}}, \quad l=1,2, \ldots, n-1$,

where $q_{l^{+}}=p_{l^{+}}^{\max }+1-\left|p_{l^{+}}^{0}\right|$ and $Q=\prod_{l=1}^{n-1} q_{l^{+}}$. Our predictions of optimal cogwheel cycles depend upon whether the set of values $\xi_{l^{+}}$do or do not all share a common prime factor.

\subsection{Category 1}

The values $\xi_{l^{+}}$do not all share a common prime factor. In this case, the optimal cogwheel cycle has a value of $N$ given by

$N_{\text {opt }}=N_{\text {pred }}$.

In addition, the optimal cogwheel winding numbers are given by

$\Delta v_{l^{+}}^{\mathrm{opt}}=\xi_{l^{+}}$.

\subsection{Category 2}

The values $\xi_{l^{+}} d o$ all share a common prime factor. In this case, we are not able to predict the value of $N$ for the optimal cogwheel cycle directly. However, we have always found that

$N_{\text {opt }} \geqslant N_{\text {pred }}$.

This property allows numerical searches to be initiated at $N_{\text {pred }}$ and proceed to higher values of $N$. We have 
usually found $N_{\text {opt }}$ to be only slightly larger than $N_{\text {pred }}$, so that in most cases, the amount of numerical effort in the search is greatly reduced. Numerical searches are necessary to discover the optimal values of $\Delta v_{l^{+}}$, in this case.

\subsection{Examples of cogwheel phase cycles selecting a single coherence pathway}

Table 1 compares values for the optimal cogwheel phase cycle length $N_{\text {opt }}$ (found by long numerical searches) with the value of $N_{\text {pred }}$ (Eq. (23)), for the selection of a single coherence pathway of two selected coherence orders in a spin system where $p_{1^{+}}^{\max }=p_{2^{+}}^{\max }=3$. Only the magnitude of the coherence order is important for the optimal length. Category 1 cases are shown in non-italic script, while Category 2 cases are shown in italics. This table shows that Eqs. (25)-(27) apply to all the cases shown and that even for Category 2 cases, the equality in Eq. (27) applies much more often than the inequality. In the table shown, the only case for which $N_{\text {opt }}>N_{\text {pred }}$ involves the selection of the pathway $\{0,2,2,-1\}$ (for which $N_{\text {opt }}=22$ while $N_{\text {pred }}=20$ ).

Table 2 compares the sets of optimal winding numbers $\Delta v_{l^{+}}^{\text {opt }}$ (found by numerical searches) with the values

Table 1

Values of $N_{\min }$ and $N_{\text {pred }}$ for the selection of a single pathway $\left\{0, p_{1^{+}}\right.$, $\left.p_{2^{+}},-1\right\}$ with $p_{1^{+}}^{\max }=p_{2^{+}}^{\max }=3$

\begin{tabular}{|c|c|c|c|c|c|c|c|c|c|c|}
\hline & & & & & & & & & & \\
\hline & & & & & & & & & & \\
\hline & 0 & 1 & 2 & 3 & & & 0 & 1 & 2 & 3 \\
\hline 0 & 16 & 20 & 24 & 28 & & 0 & 16 & 20 & 24 & 28 \\
\hline 1 & 20 & 21 & 22 & 23 & $p$ & 1 & 20 & 21 & 22 & 23 \\
\hline 2 & 24 & 22 & 22 & 18 & & 2 & 24 & 22 & 20 & 18 \\
\hline 3 & 28 & 23 & 18 & 13 & & 3 & 28 & 23 & 18 & 13 \\
\hline
\end{tabular}

Values in italics are cases which fall into Category 2, non-italics indicate Category 1 cases.

Table 2

Values of $\left\{\Delta v_{1^{+}}^{\text {opt }}, \Delta v_{2^{+}}^{\text {opt }}\right\}$ and $\left\{\xi_{1^{+}}, \xi_{2^{+}}\right\}$for the selection of a single pathway $\left\{0, p_{1^{+}}, p_{2^{+}},-1\right\}$ with $p_{1^{+}}^{\max }=p_{2^{+}}^{\max }=3$

\begin{tabular}{|c|c|c|c|c|c|c|c|c|c|c|}
\hline & \multicolumn{4}{|c|}{$\Delta v_{1^{+}}^{\mathrm{opt}}, \Delta v_{2^{+}}^{\mathrm{opt}}$} & & & \multicolumn{4}{|c|}{$\xi_{1^{+}}, \xi_{2^{+}}$} \\
\hline & \multicolumn{4}{|c|}{$p_{z^{+}}$} & & & \multicolumn{4}{|c|}{$p_{z^{+}}$} \\
\hline & 0 & 1 & 2 & 3 & & & 0 & 1 & 2 & 3 \\
\hline 0 & $\{4,1\}$ & $\{3,4\}$ & $\{1,4\}$ & $\{1,4\}$ & & 0 & $\{4,4\}$ & $\{3,4\}$ & $\{2,4\}$ & $\{1,4\}$ \\
\hline 1 & $\{4,3\}$ & $\{5,-2\}$ & $\{2,3\}$ & $\{1,3\}$ & $p$ & 1 & $\{4,3\}$ & $\{3,3\}$ & $\{2,3\}$ & $\{1,3\}$ \\
\hline 2 & $\{4,1\}$ & $\{3,2\}$ & $\{6,-5\}$ & $\{1,2\}$ & & 2 & $\{4,2\}$ & $\{3,2\}$ & $\{2,2\}$ & $\{1,2\}$ \\
\hline 3 & $\{4,1\}$ & $\{3,1\}$ & $\{2,1\}$ & $\{1,1\}$ & & 3 & $\{4,1\}$ & $\{3,1\}$ & $\{2,1\}$ & $\{1,1\}$ \\
\hline
\end{tabular}

Values in italics are cases for which the values of $\xi_{1^{+}}$and $\xi_{2^{+}}$share a common prime factor and hence fall into Category 2, non-italics indicate Category 1 cases. $\xi_{l^{+}}$given in Eq. (24), for the same pathway selections given in Table 1. For all of the Category 1 cases, $\Delta v_{l^{+}}^{\text {opt }}$ and $\xi_{l^{+}}$match exactly, as predicted by Eq. (26). For the Category 2 cases, on the other hand, $\Delta v_{l^{+}}^{\text {opt }}$ and $\xi_{l^{+}}$do not correspond. However, even in the Category 2 cases, the constraint given by Eq. (27) allows the winding numbers to be discovered by a relatively brief numerical search.

Tables 1 and 2 are only small extracts from numerical evaluations for more than 30,000 pathway selection tasks, involving all possible single-pathway selections with $n=3$ and 4 and with values of $p_{l^{+}}^{\max }$ up to 10 . Some cases with $n>4$ have also been investigated. We did not find a single case in conflict with Eqs. (25)-(27).

\subsection{Selecting two coherence pathways, $p_{l^{+}}^{0}=-p_{l^{+}}^{0^{\prime}}$}

It has been found that the case where $p_{l^{+}}^{0}=-p_{l^{+}}^{0^{\prime}}$ for all selected coherence orders (except, of course, the last which is always -1) is closely related to the case of selecting a single coherence pathway. Consider the quantity

$N_{\text {pred }}=\sum_{l=1}^{n-1}\left(\frac{2 Q\left|p_{l^{+}}^{0}\right|}{q_{l^{+}}}\right)$,

together with the values of $\xi_{l^{+}}$from Eq. (24). Our predictions of optimal cogwheel cycles again depend upon whether or not the set of $\xi_{l^{+}}$values all share a common prime factor. If they do not, the cycle falls into Category 1 stated above, using the definition of $N_{\text {pred }}$ given in Eq. (28). If they do, the cycle falls into Category 2 and numerical searches, starting at $N_{\text {pred }}$, are required.

\section{Examples}

The split- $t_{1} 5 \mathrm{Q} 3 \mathrm{QMAS}$ experiment [8] on spins $I=5 / 2$ consists of four RF blocks with selection of the coherence pathway $\{0,+5,+3,+1,-1\}$. To select this coherence pathway using nested phase cycling as described above would require 693 steps (Eq. (9)). This can be done in several ways. One would be to cycle the first RF block in 11 steps, selecting a change of +5 , cycle the second in 9 steps, selecting a change of -2 , and cycle the third in 7 steps, also selecting a change of -2 . In fact, the original implementation [8] used a 640 step phase cycle, already illustrating the potential redundancy within nested phase cycles. Eq. (23) can be used to predict the length of the optimum cogwheel phase cycle. The values to be inserted are $\left\{p_{1^{+}}^{0}, p_{2^{+}}^{0}, p_{3^{+}}^{0}\right\}=\{5,3,1\},\left\{q_{1^{+}}, q_{2^{+}}, q_{3^{+}}\right\}=\{1,3,5\}$, and $Q=15$. This gives a predicted phase-cycle length of 201. Furthermore, using the same values, Eqs. (24) and (26) give values of $\left\{\Delta v_{1^{+}}^{\mathrm{opt}}, \Delta v_{2^{+}}^{\mathrm{opt}}, \Delta v_{3^{+}}^{\mathrm{opt}}\right\}=\{15,5,3\}$. As these do not share a common prime factor, a cogwheel phase cycle based upon these values is predicted to be selective for the desired coherence order selection. This 
may be confirmed by computer search. One such phase cycle is $\operatorname{COG} 201(0,15,20,23 ; 116)$.

A variety of NMR experiments, including TOSS and PASS, require the selection of coherence pathways consisting of alternating +1 and -1 coherence orders whilst assuming that no higher coherence orders may occur. These require very long nested phase cycles. Cogwheel phase cycles are, on the other hand, much shorter for these cases [4]. Using the method discussed earlier would suggest nested phase cycles of length $3^{k}$. In fact, it is possible to only cycle every other RF block to within a nested phase cycle. Thus, for an even number of conversions, $k$, between +1 and -1 coherence orders, nested phase cycles can be constructed with $5^{k / 2}$ steps, whilst for a odd number of conversions, nested phase cycles can be constructed with $3 \times 5^{(k-1) / 2}$ steps. From Eq. (23), the predicted length of a cogwheel phase cycle for such an experiment is given by $2 k+1$. Eqs. (24) and (26) give values of $\left\{\Delta v_{1^{+}}^{\text {pred }}, \Delta v_{2^{+}}^{\text {pred }}, \ldots, \Delta v_{n-1^{+}}^{\text {pred }}, \Delta v_{n^{+}}^{\text {pred }}\right\}$ which alternate between +1 and -1 . As these share no prime factors, a cogwheel phase cycle based upon these values is predicted to work successfully. This phase cycle can also be predicted using the diagrams discussed in [4]. These predictions have been verified experimentally for $k=5$, where the conversions between +1 and -1 coherence orders can be selected with $\operatorname{COG} 11(0,1,0,1,0,1 ; 6)$.

A third example has also been successfully implemented [9] to acquire a double-quantum spin echo in a solid-state MAS experiment on ${ }^{13} \mathrm{C}$ nuclei. The experiment consists of five RF blocks, with selection of two coherence pathways, $\{0,+2,-2,+2,0,-1\}$ and $\{0,-2,+2$, $-2,0,-1\}$ in a system of two spins $I=1 / 2$, where the maximum and minimum possible coherences are assumed to be \pm 2 throughout. Fig. 2A shows a schematic of the pulse sequence. The first RF block is a sequence such as SC14 [10] for the excitation of double-quantum coherence. This is followed by two $180^{\circ}$ pulses (blocks 2 and 3 ), one placed in the middle of $t_{1}$ and one at the end of $t_{1}$. The first refocuses chemical shifts whilst the second returns the magnetization to the coherence orders it possessed at the beginning of $t_{1}$. The fourth RF block is again a sequence such as $\mathrm{SC} 14$ for the reconversion of doublequantum coherence to longitudinal magnetization and the fifth $\mathrm{RF}$ block is a $90^{\circ}$ pulse to generate $(-1)$-quantum coherence for detection in $t_{2}$. Fig. 2B shows the coherence transfer pathway diagram.

A nested phase cycle would take 1024 transients. Inserting the values $\left\{p_{1^{+}}^{0}, p_{2^{+}}^{0}, p_{3^{+}}^{0}, p_{4^{+}}^{0}\right\}=\{+2,-2,+2,0\}$, $\left\{q_{1^{+}}, q_{2^{+}}, q_{3^{+}}, q_{4^{+}}\right\}=\{1,1,1,3\}$, and $Q=3$ into Eqs. (24) and (28) gives $N_{\text {pred }}=36$ and $\left\{\xi_{1^{+}}, \xi_{2^{+}}, \xi_{3^{+}}, \xi_{4^{+}}\right\}=$ $\{+3,-3,+3,+1\}$. Since the values of $\xi_{l^{+}}$do not share a common prime factor, a successful cogwheel phase can be constructed with $N_{\mathrm{opt}}=36$ and $\left\{\Delta v_{1^{+}}, \Delta v_{2^{+}}, \Delta v_{3^{+}}, \Delta v_{4^{+}}\right\}$ $=\{+3,-3,+3,+1\}$. One such phase cycle is COG36(0,3,0,3,4;22). This has the same selectivity as the nested phase cycle in just $3.5 \%$ the number of tran-
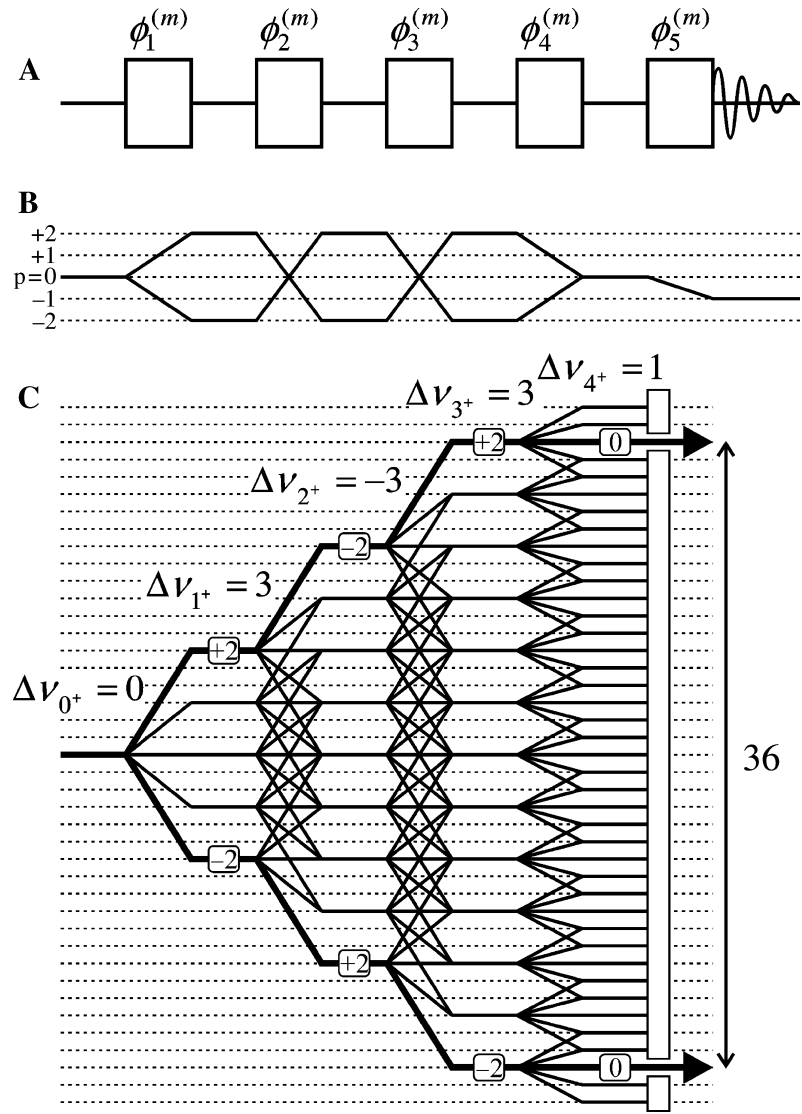

Fig. 2. (A) Schematic pulse sequence for a double-quantum spin echo in the context of a solid-state MAS experiment, as described in the text. The RF blocks are labelled 1-5. (B) Coherence selection pathway diagram for a double-quantum spin echo. Two pathways are selected. (C) The cogwheel selection diagram for selecting these coherence pathways. Starting from the left, the lines represent the accumulated phase acquired by a pathway, given by Eq. (8). The two thick lines represent the desired pathways and the barrier on the right has holes separated by 36 units, allowing through only the desired pathways.

sients. Fig. $2 \mathrm{C}$ shows the cogwheel selection diagram for this phase cycle, including the values of $\Delta v_{l^{+}}$.

\section{Conclusions}

The predictions set out in this paper have been tested extensively. In some predictable situations, these rules lead immediately to the optimal cogwheel winding numbers. In other cases, the rules greatly reduce the time needed to discover the optimal cycle by numerical searches. However, they lack proofs. Attempts have been made and continue to be made in order to understand the origin of these equations.

\section{References}

[1] M.H. Levitt, P.K. Madhu, C.E. Hughes, Cogwheel phase cycling, J. Magn. Reson. 155 (2002) 300-306. 
[2] G. Bodenhausen, H. Kogler, R.R. Ernst, Selection of coherence transfer pathways in NMR pulse experiments, J. Magn. Reson. 58 (1984) 370-388.

[3] A.D. Bain, Coherence levels and coherence pathways in NMR. A simple way to design phase cycling procedures, J. Magn. Reson. 56 (1984) 418-427.

[4] N. Ivchenko, C.E. Hughes, M.H. Levitt, Application of cogwheel phase cycling to sideband manipulation experiments in solid-state NMR, J. Magn. Reson. 164 (2003) 286-293.

[5] A. Jerschow, N. Müller, Efficient simulation of coherence transfer pathway selection by phase cycling and pulsed field gradients in NMR, J. Magn. Reson. 134 (1998) 17-29.

[6] A. Jerschow, R. Kumar, Calculation of coherence pathway selection and cogwheel cycles, J. Magn. Reson. 160 (2003) 5964.
[7] J. Ollerenshaw, R.E.D. McClung, Construction of phase cycles of minimum cycle length: MakeCycle, J. Magn. Reson. 143 (2000) 255-265.

[8] J.W. Logan, J.T. Urban, J.D. Walls, K.H. Lim, A. Jerschow, A. Pines, Investigations of low-amplitude radio frequency pulses at and away from rotary resonance conditions for $I=5 / 2$ nuclei, Solid State Nucl. Magn. Reson. 22 (2002) 97-109.

[9] C.E. Hughes, J. Schmedt auf der Günne, M.H. Levitt, A test for the number of coupled spins $I=1 / 2$ in magic-angle spinning solids. Zero-quantum recoupling of multiple-quantum coherences, ChemPhysChem. 4 (2003) 457-465.

[10] A. Brinkmann, M. Edén, M.H. Levitt, Synchronous helical pulse sequences in magic-angle spinning NMR. Double quantum recoupling of multiple-spin systems, J. Chem. Phys. 112 (2000) $8539-8554$. 